(c) Н.О. Ховасова ${ }^{1,2}$ * А.В. Наумов ${ }^{1,2}$, О.Н. Ткачева ${ }^{1,2}$, Е.Н. Дудинская ${ }^{1,2}$

'Кафедра болезней старения Российского национального исследовательского медицинского университета им. Н.И. Пирогова, Москва, Россия

²Российский геронтологический научно-клинический центр, Москва, Россия

ОБОСНОВАНИЕ. Пожилые люди с остеопорозом (ОП) и высоким риском падений - наиболее уязвимая группа пациентов в отношении развития переломов. Падения и переломы у пожилых пациентов с ОП ассоциированы с гериатрическими синдромами и худшим функциональным статусом.

ЦЕЛЬ. Оценить коморбидный и гериатрический статус пациентов пожилого и старческого возраста с ОП и без него.

МАТЕРИАЛЫ И МЕТОДЫ. В исследование включены 607 пациентов старше 60 лет, госпитализированных в гериатрическое отделение. По наличию ОП пациенты были разделены на 2 группы: 1 группа - пациенты с ОП (n=178, 29,3\%),

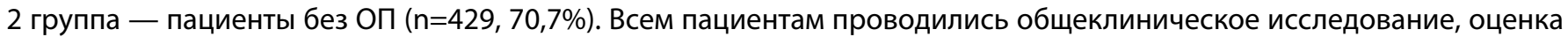
коморбидности по индексу Чарльсон, комплексная гериатрическая оценка.

РЕЗУЛЬтАТЫ. ОП имели 178 (29,3\%) пациентов, чаще это были женщины. 55,6\% пациентов с ОП были инвалидами. У пациентов с ОП достоверно чаще встречались возраст-ассоциированные заболевания - болезнь Альцгеймера, болезнь Паркинсона, остеоартрит, анемия, заболевания щитовидной железы, варикозная болезнь. Практически со всеми этими заболеваниями при однофакторном анализе выявлена ассоциация с ОП. У пациентов 1 группы достоверно чаще встречались такие гериатрические синдромы, как старческая астения, гиподинамия, недостаточность питания, полипрагмазия, недержание мочи. Пациенты с ОП чаще проживали одиноко и использовали вспомогательные средства при передвижении в сравнении с пациентами без ОП. Проведенный однофакторный анализ продемонстрировал, что ОП ассоциирован (отношение шансов (ОШ) 1,54-2,00) со старческой астенией, гиподинамией, использованием вспомогательных средств при передвижении, нарушением сна, сенсорным дефицитом по зрению, недержанием мочи. Функциональный статус пациентов с ОП был хуже по сравнению с пациентами без ОП. Пациенты с ОП перенесли больше переломов.

ЗАКЛЮЧЕНИЕ. Пациенты с ОП имеют высокую коморбидность, отягощенный гериатрический статус. У пациентов пожилого и старческого возраста необходимо не только проводить скрининг и диагностику ОП, оценивать риск 10-летней вероятности основных патологических переломов по алгоритму FRAX, но и проводить комплексную гериатрическую оценку для диагностики гериатрических синдромов, которые утяжеляют течение ОП и приводят к более серьезным последствиям.

КЛЮЧЕВЫЕ СЛОВА:остеопороз; падения; переломы; гериатрический синдром.

\title{
CHARACTERISTICS OF GERIATRIC AND SOMATIC STATUS IN PATIENTS WITH OSTEOPOROSIS
}

(C) Natalia O. Khovasova ${ }^{1,2 *}$, Anton V. Naumov ${ }^{1,2}$, Olga N. Tkacheva' ${ }^{1}$, Ekaterina N. Dudinskaya ${ }^{1,2}$

'Department of diseases of aging of The Pirogov Russian National Research Medical University, Moscow, Russia ${ }^{2}$ Russian Gerontology Clinical Research, Moscow, Russia

BACKGROUND: Older adults with osteoporosis (OP) and high risk of falls are the most vulnerable group of patients with respect to the development of fractures. Falls and fractures in elderly patients with OP are associated with geriatric syndromes and worse functional status.

AIM: To assess comorbidity and geriatric status in elderly and senile patients with and without OP.

MATERIALS AND METHODS: The study included 607 patients over 60 years of age hospitalized in the geriatric department. According to the presence of OP, the patients were divided into 2 groups: group 1 - patients with OP ( $n=178,29.3 \%$ ), group 2 - patients without OP $(n=429,70.7 \%)$. All patients underwent a general clinical study, an assessment of comorbidity according to the Charlson index, and a comprehensive geriatric score.

RESULTS: OPs had 178 (29.3\%) patients, more often these were women. 55.6\% of patients with OP were disabled. Agerelated diseases such as Alzheimer's disease, Parkinson's disease, osteoarthritis, anemia, thyroid disease, varicose veins were 
significantly more common in patients with OP. With almost all of these diseases, a univariate analysis revealed an association with OP. Geriatric syndromes such as frailty, hypodynamia, malnutrition, polypharmacy, urinary incontinence were significantly more common in group 1 patients. Patients with OP were more likely to live alone and use mobility aids compared to patients without OP.

The univariate analysis demonstrated that OP is associated (OR 1.54 to 2.00) with frailty, hypodynamia, the use of aids in movement, sleep disorders, sensory vision deficiency, urinary incontinence. The Functional status of patients with OP was worse compared to patients without OP. Patients with OP suffered more fractures, and vertebral fractures were significantly more frequent.

CONCLUSION: Patients with OP have a high comorbidity, a burdened geriatric status. In elderly patients, it is necessary not only to screen and diagnose OP, to assess the risk of 10-years probability of major pathological fractures using the FRAX algorithm, but also to conduct a comprehensive geriatric assessment to diagnose geriatric syndromes that weaken the course of OP and lead to more serious consequences.

KEYWORDS: osteoporosis; falls; fractures; geriatric syndrome.

\section{ОБОСНОВАНИЕ}

Остеопороз (ОП) - системное заболевание костной ткани, характеризующееся снижением костной массы и нарушением микроархитектоники кости [1]. Диагностические критерии ОП четко определены.

1. Наличие патологических переломов крупных костей скелета в анамнезе или выявленных при обследовании, независимо от результатов рентгеноденситометрии или FRAX.

2. Наличие высокой индивидуальной 10-летней вероятности основных патологических переломов независимо от показателя денситометрии.

3. Снижение минеральной плотности кости (МПК) на 2,5 и более стандартных отклонений по Т-критерию в шейке бедренной кости, и/или в целом в проксимальном отделе бедренной кости, и/или в поясничных позвонках, измеренной двухэнергетической рентгеноденситометрией, у женщин в постменопаузе и мужчин старше 50 лет [2].

ОП и остеопоротические переломы длительное время ассоциировались с женщинами в постменопаузальном периоде. Однако в современной реальности частота переломов растет из-за старения населения. В США сегодня ОП встречается у 46,3\% мужчин и 77,1\% женщин пожилого и старческого возраста [3]. В странах Западной Европы риск любого остеопоротического перелома в течение жизни составляет 40-50\% для женщин и 13-22\% для мужчин [4].

К 2050 г. в мире прогнозируется увеличение лиц пожилого и старческого возраста примерно в 2 раза. Однако увеличение продолжительности жизни не равно увеличению продолжительности здоровой и качественной жизни. Добавленные к жизни годы зачастую характеризуются заболеваниями и инвалидностью [5]. Согласно докладу Международного фонда по ОП, к 2040 г. число пациентов с ОП в возрасте старше 50 лет удвоится по сравнению с показателями 2010 г. [6]. В связи с этим вмешательства по снижению частоты новых случаев, а также прогрессирования уже имеющегося ОП и предотвращения переломов должны быть сосредоточены на пожилой категории населения [7].

Тяжелые медицинские, социальные и экономические последствия при ОП связаны прежде всего с низкоэнергетическими переломами, приводящими к хронической боли, депрессии, потере автономности, инвалидиза- ции и смерти. Повышенная смертность после перелома проксимального отдела бедра в значительной степени зависит от возраста и выше у мужчин. Но в популяционном масштабе важно и то, что статистически значимая повышенная смертность сохраняется в течение 10 лет после случившегося перелома шейки бедра [8].

Одним из провоцирующих факторов переломов являются падения. Центры по контролю и профилактике заболеваний в США сообщают, что в 2014 г. почти 30\% пожилых людей сообщили о падении. Возраст сам по себе является фактором риска падений: частота падений в 90 лет в 2 раза выше, чем в 60 лет [9]. Другие признанные факторы риска падений - это остеоартрит, депрессия, сердечно-сосудистые заболевания и недержание мочи [10].

Падения у пожилых пациентов с ОП, имеющих более высокий риск переломов, приводят к нарастанию нуждаемости в посторонней помощи, увеличению частоты госпитализаций и инвалидизации [11]. Поэтому пациенты с низкой МПК и высоким риском падений - наиболее уязвимая группа пожилых людей в отношении развития переломов. Риск падений и переломов у пожилых пациентов с ОП ассоциирован с гериатрическими синдромами - саркопенией, полиморбидностью и старческой астенией (СА) [12].

СА и ОП имеют общие факторы риска - возраст, низкая физическая активность, потеря массы тела, когнитивные нарушения. И до сих пор однозначно неясно, что первично - ОП или СА [13]. Ряд исследований демонстрирует связь тяжести ОП и риска переломов у пациентов со СА. Так, у женщин с синдромом СА риск переломов шейки бедра (отношение шансов (ОШ) 1,40; 95\% доверительный интервал (ДИ) 1,03-1,90) более высокий, чем у крепких женщин того же возраста [14]. С другой стороны, СА имеет более тяжелое течение у женщин, которые перенесли остеопоротический перелом, что свидетельствует о большем накоплении дефицитов и ускорении прогрессирования СА после остеопоротического перелома [15]. В ряде исследований было установлено, что комплексная оценка по диагностике СА сопоставима c FRAX при прогнозировании риска основных остеопоротических переломов и переломов проксимального отдела бедра, что указывает на то, что оценка наличия СА может определять риск переломов у пожилых людей. В связи с чем сегодня обсуждается возможность интеграции параметров, характеризующих CA, в шкалу FRAX для более точного прогнозирования риска переломов [16]. 
Таблица 1. Общая характеристика пациентов

\begin{tabular}{lcccc}
\hline & С ОП & \% & Без ОП & \% \\
\hline Количество & 178 & 29,3 & 429 & 70,7 \\
Возраст & $77,1 \pm 7,39$ & & $74,3 \pm 8,01$ & $77,9^{*}$ \\
Женщины & 159 & 89,3 & 334 & 95 \\
Мужчины & 19 & 10,7 & 164 & $37,4^{*}$ \\
Инвалидность & 99 & 55,6 & & \\
\hline
\end{tabular}

* $p<0,05$.

Ряд исследований показывает связь между низкой МПК и саркопенией как у мужчин, так и у женщин пожилого возраста [17-19]. Locquet и соавт. показали на 232 пациентах старше 75 лет, что снижение мышечной функции связано с изменением микроархитектоники костей, а пациенты с саркопенией имеют 5-кратный повышенный риск развития ОП [20]. Сочетание саркопении и ОП (остеосаркопения) еще более повышает риск падений (ОШ 1,41; 95\% ДИ 1,02-1,95) и остеопоротических переломов (ОШ 1,87; 95\% ДИ 1,07-3,26) по сравнению с пациентами, не имеющими ни ОП, ни саркопении [21-23]. Кости и мышцы тесно взаимодействуют друг с другом не только анатомически, но и метаболически. Например, имеется перекрестная связь «мышца-кость» за счет факторов, синтезируемых мышцей, - инсулиноподобного фактора роста-1, фактора роста фибробластов, ИЛ-6, ИЛ-15, миостатина, остеоактивина, которые В том числе влияют на моделирование кости [24] и прогрессирование ОП [25]. Также нельзя забывать о значении хронического воспаления, являющегося следствием совокупного действия антигенной нагрузки в течение всей жизни, вызванной как клиническими, так и субклиническими инфекциями, а также воздействием неинфекционных антигенов. Следствием этого являются воспалительный ответ, повреждение тканей, высвобождение дополнительных цитокинов, что определяет порочный круг, приводящий к хроническому провоспалительному состоянию - inflammaging [26]. Системное воспаление, саркопения, ОП приводят к нездоровому старению, характеризующемуся развитием и прогрессированием гериатрических синдромов. С этой точки зрения представляется интересным охарактеризовать гериатрический портрет пациента с ОП.

\section{ЦЕЛЬ ИССЛЕДОВАНИЯ}

Оценить коморбидный и гериатрический статус пациентов пожилого и старческого возраста с ОП и без него.

\section{МАТЕРИАЛЫ И МЕТОДЫ}

Место и время проведения исследования

Исследование проводилось на базе ОСП «Российский геронтологический научно-клинический центр» ФГАОУ ВО РНИМУ им. Н.И. Пирогова Минздрава России, Москва, в период июнь 2019-февраль 2021 г.

\section{Изучаемые популяции}

Пациенты (n=607) старше 60 лет, госпитализированные в гериатрическое отделение. Критерии включения: возраст 60 лет и старше; согласие на участие в исследовании. Критерии исключения: возраст менее 60 лет; онкологические заболевания; тяжелая почечная недостаточность; тяжелая печеночная недостаточность; отказ от участия в исследовании.

Способ формирования выборки из изучаемой популяции: сплошной.

\section{Дизайн исследования}

Одноцентровое одномоментное наблюдательное исследование. По наличию ОП пациенты были разделены на 2 группы: 1 группа - пациенты с ОП (критерии: наличие патологических переломов костей скелета в анамнезе или выявленных при обследовании; высокая (для лиц 60-89 лет-— более $20,12 \%$, а для лиц 90 - более $18,8 \%$ ) индивидуальная 10-летняя вероятность основных патологических переломов по алгоритму FRAX; снижение МПК на 2,5 стандартных отклонения и более по Т-критерию в шейке бедренной кости и/или в целом в проксимальном отделе бедренной кости и/или в поясничных позвонках), 2 группа - пациенты без ОП. Общая характеристика пациентов представлена в табл. 1.

\section{Методы}

ОП диагностировался согласно диагностическим критериям: 1) наличие низкоэнергетических переломов крупных костей скелета в анамнезе или выявленных при обследовании, независимо от результатов рентгеноденситометрии или FRAX; 2) наличие высокой индивидуальной 10-летней вероятности основных низкоэнергетических переломов независимо от показателя денситометрии; 3) снижение МПК на 2,5 стандартных отклонения и более по Т-критерию в шейке бедренной кости, и/или в целом в проксимальном отделе бедренной кости, и/или в поясничных позвонках, измеренной двухэнергетической рентгеноденситометрией, у женщин в постменопаузе и мужчин старше 50 лет [2]. Для оценки коморбидного статуса всем пациентам было проведено общеклиническое исследование, рассчитан индекс коморбидности Чарльсон. Для характеристики гериатрического статуса всем пациентам проведена комплексная гериатрическая оценка (КГО) согласно методологии, описанной в российских клинических рекомендациях «Старческая астения» [27]. Оценку МПК проводили методом двухэнергетической рентгеновской абсорбциометрии с помощью аппарата Prodigy, GE Lunar (DXA). Измерялись суммарная МПК поясничного отдела позвоночника $\left(\mathrm{L}_{1}-\mathrm{L}_{\mathrm{IV}}\right), \mathrm{MПК} \mathrm{области} \mathrm{шейки} \mathrm{бедренной} \mathrm{кости,} \mathrm{суммарная}$ МПК бедренной кости. Денситометрическое исследование проводилось одним специалистом. 
Статистический анализ

База данных создана в программе IBM ${ }^{\circledR}$ SPSS $^{\circledR}$ Statistics version 23.0 (SPSS Inc., США). Вид распределения количественных переменных анализировали при помощи одновыборочного критерия Колмогорова-Смирнова. Количественные показатели приведены в виде среднего арифметического (М) с соответствующим стандартным отклонением (SD). Качественные данные представлены в виде абсолютных чисел и относительных частот. Для ненормально распределенных показателей применялся непараметрический критерий Манна-Уитни, для нормально распределенных - Т-критерий Стьюдента. Взаимосвязи между переменными оценивали при помощи однофакторного анализа, для чего использовали бинарную логистическую регрессию с вычислением отношения шансов (ОШ) и 95\% доверительного интервала (ДИ). Корреляционный анализ проводили с использованием ранговой корреляции Спирмена. Различия считали значимыми при $\mathrm{p}<0,05$.

\section{Этическая экспертиза}

Протокол исследования рассмотрен и одобрен независимым этическим комитетом ОСП Российский геронтологический научно-клинический центр ФГАОУ ВО РНИМУ им. Н.И. Пирогова (протокол заседания № 25 от 17.06.2019).

\section{РЕЗУЛЬТАТЫ}

Каждый третий пациент (29,3\%) старше 60 лет страдал ОП, чаще это были женщины. У 96 (53,9\%) человек ОП был подтвержден данными денситометрии, у 40 (22,5\%) высокой индивидуальной 10-летней вероятностью основных низкоэнергетических переломов, у 75 (42,1\%) наличием низкоэнергетических переломов костей скелета в анамнезе или выявленных при рентгенографии грудного и поясничного отделов позвонков, выполненной в двух проекциях. Необходимо отметить, что у 10 пациентов при наличии низкоэнергетических переломов в анамнезе на амбулаторном этапе диагноз ОП не выставлялся и не проводилось никакой антиостеопоротической терапии.

$55,6 \%$ пациентов с ОП имели инвалидность, что в 1,5 раза чаще, чем у пациентов без него (табл. 1). Это, в том числе, можно объяснить более высокой распространенностью коморбидности у пациентов с ОП (табл. 2).

Полученные данные демонстрируют, что у пациентов 1 группы достоверно чаще встречались заболевания щитовидной железы, болезнь Альцгеймера, болезнь Паркинсона, анемия, остеоартрит, варикозная болезнь. Индекс коморбидности Чарльсон был выше также в 1 группе наблюдения. Далее при помощи однофакторного анализа была изучена взаимосвязь между ОП и соматическими заболеваниями. Выявлены ассоциации ОП с заболеваниями щитовидной железы, остеоартритом, анемией, варикозной болезнью вен и болезнью Паркинсона. С другими заболеваниями достоверной ассоциации выявлено не было (табл. 3).

При проведении комплексной гериатрической оценки были диагностированы различные гериатрические синдромы, структура которых представлена в табл. 4.

Таблица 2. Распространенность и структура коморбидной патологии

\begin{tabular}{|c|c|c|c|c|c|}
\hline & $\begin{array}{c}\text { C ОП } \\
(n=178)\end{array}$ & $\%$ & $\begin{array}{c}\text { Без ОП } \\
(n=429)\end{array}$ & $\%$ & $\mathbf{p}$ \\
\hline Индекс коморбидности Чарльсон & $6,04 \pm 1,72$ & & $5,52 \pm 2,02$ & & $<0,001$ \\
\hline $\mathrm{A} \Gamma$ & 172 & 96,6 & 413 & 96,2 & 0,414 \\
\hline ИБС & 84 & 47,2 & 195 & 45,5 & 0,343 \\
\hline ФП & 33 & 18,5 & 84 & 19,6 & 0,383 \\
\hline $\mathrm{XCH}$ & 62 & 34,8 & 135 & 31,5 & 0,210 \\
\hline OНМК, ТИА & 26 & 14,6 & 58 & 13,5 & 0,442 \\
\hline СД 2 типа & 45 & 25,3 & 107 & 24,9 & 0,462 \\
\hline Заболевания щитовидной железы & 67 & 37,6 & 112 & 26,1 & 0,002 \\
\hline Ожирение & 76 & 44,7 & 199 & 46,4 & 0,212 \\
\hline Болезнь Альцгеймера & 4 & 2,2 & 2 & 0,5 & 0,021 \\
\hline Болезнь Паркинсона & 12 & 5,6 & 10 & 2,3 & 0,002 \\
\hline Анемия & 34 & 19,1 & 51 & 11,9 & 0,019 \\
\hline ХОБЛ & 27 & 15,2 & 59 & 13,8 & 0392 \\
\hline Мочекаменная болезнь & 28 & 15,7 & 64 & 14,9 & 0,244 \\
\hline Язвенная болезнь желудка и двенадцатиперстной кишки & 34 & 19,1 & 73 & 17 & 0,275 \\
\hline Варикозная болезнь & 86 & 48,3 & 170 & 39,6 & 0,024 \\
\hline Остеоартрит & 144 & 80,9 & 220 & 51,3 & 0,005 \\
\hline Подагра & 9 & 5,3 & 20 & 4,7 & 0,418 \\
\hline Доброкачественная гиперплазия предстательной железы & 13 & 68,4 & 49 & 51,6 & 0,096 \\
\hline
\end{tabular}

Примечание к табл. 2 и 3. АГ - артериальная гипертензия; ИБС - ишемическая болезнь сердца; ФП — фибрилляция предсердий; ХСН - хроническая сердечная недостаточность; ОНМК, ТИА — острое нарушение мозгового кровообращения или транзиторная ишемическая атака; ХОБЛ хроническая обструктивная болезнь легких. Курсивом указаны статистически достоверные результаты. 
Таблица 3. Ассоциации между остеопорозом и коморбидными заболеваниями

\begin{tabular}{|c|c|c|c|}
\hline & ОШ & 95\% ди & $\mathbf{p}$ \\
\hline Остеоартрит & 4,02 & $2,65-6,11$ & $<0,05$ \\
\hline Болезнь Паркинсона & 3,03 & $1,28-7,16$ & $<0,05$ \\
\hline Заболевания щитовидной железы & 1,71 & $1,18-2,48$ & $<0,05$ \\
\hline Анемия & 1,71 & $1,06-2,74$ & $<0,05$ \\
\hline Варикозная болезнь & 1,42 & $1,01-2,03$ & $<0,05$ \\
\hline $\mathrm{A} \Gamma$ & 1,11 & $0,49-2,89$ & $>0,05$ \\
\hline ИБС & 1,07 & $0,76-1,52$ & $>0,05$ \\
\hline ФП & 0,94 & $0,60-1,46$ & $>0,05$ \\
\hline $\mathrm{XCH}$ & 1,16 & $0,80-1,68$ & $>0,05$ \\
\hline OHМК+ТИА & 1,09 & $0,66-1,80$ & $>0,05$ \\
\hline СД 2 типа & 1,02 & $0,68-1,52$ & $>0,05$ \\
\hline Ожирение & 0,86 & $0,60-1,22$ & $>0,05$ \\
\hline Болезнь Альцгеймера & 4,91 & $0,89-27,04$ & $>0,05$ \\
\hline ХОБЛ & 1,12 & $0,68-1,84$ & $>0,05$ \\
\hline Мочекаменная болезнь & 1,07 & $0,66-1,73$ & $>0,05$ \\
\hline Язвенная болезнь желудка и двенадцатиперстной кишки & 1,15 & $0,73-1,81$ & $>0,05$ \\
\hline Подагра & 1,09 & $0,49-2,44$ & $>0,05$ \\
\hline Доброкачественная гиперплазия предстательной железы & 2,03 & $0,71-5,79$ & $>0,05$ \\
\hline
\end{tabular}

Таблица 4. Распространенность и структура гериатрических синдромов

\begin{tabular}{|c|c|c|c|c|c|}
\hline & $\begin{array}{c}\text { C ОП } \\
(n=178,29,3 \%)\end{array}$ & $\%$ & $\begin{array}{c}\text { Без ОП } \\
(n=429,70,7 \%)\end{array}$ & $\%$ & $\mathbf{p}$ \\
\hline Количество гериатрических синдромов & $6,83 \pm 3,0$ & & $5,52 \pm 2,64$ & & 0,028 \\
\hline Старческая астения & 50 & 28,1 & 70 & 16,3 & $<0,001$ \\
\hline Недостаточность питания & 7 & 3,9 & 8 & 1,8 & $<0,001$ \\
\hline Хроническая боль & 82 & 46,1 & 184 & 42,9 & 0,392 \\
\hline Падения & 93 & 52,2 & 217 & 50,6 & 0,355 \\
\hline Депрессия & 20 & 11,2 & 85 & 19,8 & 0,028 \\
\hline Когнитивные нарушения (шкала MMSE, баллы) & $27,2 \pm 4,1$ & & $27,8 \pm 4,1$ & & 0,239 \\
\hline Нарушения сна & 85 & 47,8 & 160 & 37,3 & 0,324 \\
\hline Нарушения зрения & 105 & 58,9 & 185 & 43,1 & 0,286 \\
\hline Нарушения слуха & 73 & 41 & 163 & 37,9 & 0,219 \\
\hline Полипрагмазия & 44 & 24,7 & 91 & 21,2 & 0,043 \\
\hline Количество принимаемых препаратов & $4,56 \pm 2,4$ & & $4,26 \pm 2,1$ & & 0,069 \\
\hline Недержание мочи & 53 & 29,7 & 87 & 20,3 & 0,003 \\
\hline Ортостатическая гипотония & 9 & 5,1 & 24 & 5,6 & 0,282 \\
\hline Дефицит витамина D & 19 & 10,6 & 46 & 10,7 & 0,253 \\
\hline Одинокое проживание & 41 & 23 & 71 & 16,6 & 0,009 \\
\hline Головокружение & 35 & 19,6 & 70 & 16,3 & 0,048 \\
\hline Гиподинамия & 103 & 57,9 & 187 & 43,6 & $<0,001$ \\
\hline Нарушения походки & 53 & 29,8 & 113 & 26,3 & 0,212 \\
\hline Нарушения равновесия & 85 & 47,7 & 216 & 50,3 & 0,256 \\
\hline $\begin{array}{l}\text { Использование вспомогательных средств } \\
\text { при передвижении }\end{array}$ & 64 & 36 & 110 & 25,6 & 0,006 \\
\hline
\end{tabular}

Примечание: курсивом указаны статистически достоверные результаты. 
Таблица 5. Ассоциации между остеопорозом и гериатрическими синдромами

\begin{tabular}{lccc}
\hline & ОШ & $\mathbf{9 5 \% ~ Д И ~}$ & p \\
\hline Старческая астения & 2,00 & $1,32-3,04$ & $<0,05$ \\
Нарушения зрения & 1,54 & $1,08-2,19$ & $<0,05$ \\
Гиподинамия & 1,77 & $1,25-2,53$ & $<, 05$ \\
Нарушения сна & 1,54 & $1,08-2,29$ & $<0,05$ \\
Использование вспомогательных средств при передвижении & 1,63 & $1,12-2,37$ & $1,12-2,48$ \\
Недержание мочи & 1,67 & $<0,05$ \\
\hline
\end{tabular}

Таблица 6. Ассоциации между тяжелым остеопорозом и гериатрическими синдромами

\begin{tabular}{lccc}
\hline & ОШ & $\mathbf{9 5 \% ~ Д И ~}$ & p \\
\hline Ортопедическая патология стопы & 4,42 & $2,54-7,68$ & $<0,05$ \\
Депрессия & 2,09 & $1,29-3,35$ & $<0,05$ \\
Тревога & 1,78 & $1,12-2,83$ & $<0,05$ \\
Старческая астения & 1,73 & $1,09-2,74$ & $<0,05$ \\
Хроническая боль & 1,10 & $1,06-1,19$ & $<0,05$ \\
\hline
\end{tabular}

Таблица 7. Оценка функционального статуса

\begin{tabular}{|c|c|c|c|}
\hline & $\begin{array}{c}\text { C OП } \\
(n=178)\end{array}$ & $\begin{array}{l}\text { Без ОП } \\
(n=429)\end{array}$ & $\mathbf{p}$ \\
\hline «Возраст не помеха», средний балл & $3,5 \pm 1,58$ & $3,0 \pm 1,57$ & $<0,001$ \\
\hline Индекс Бартела, средний балл & $88,5 \pm 14,1$ & $93,4 \pm 9,7$ & 0,002 \\
\hline Индекс Лоутона, средний балл & $6,6 \pm 1,8$ & $7,17 \pm 1,56$ & 0,002 \\
\hline Скорость ходьбы, м/с & $0,58 \pm 0,37$ & $0,76 \pm 0,34$ & 0,011 \\
\hline Время выполнения теста «Встань и иди», с & $11,6 \pm 6,7$ & $11,3 \pm 8,5$ & 0,382 \\
\hline Суммарный балл за выполнение тандемных тестов & $2,62 \pm 1,5$ & $2,9 \pm 1,7$ & 0,181 \\
\hline $\begin{array}{l}\text { Суммарный балл по краткой батарее тестов физического } \\
\text { функционирования }\end{array}$ & $7,4 \pm 3,3$ & $7,19 \pm 3,2$ & 0,228 \\
\hline Пациенты со снижением мышечной силы рук, n (\%) & $33(18,5)$ & $131(30,5)$ & 0,331 \\
\hline Время, необходимое для 5 подъемов со стула, с & $16,5 \pm 10,3$ & $13,5 \pm 9,5$ & 0,003 \\
\hline
\end{tabular}

Примечание: курсивом указаны статистически достоверные результаты.

Согласно полученным данным, количество гериатрических синдромов, диагностированных у одного пациента, было выше у пациентов 1 группы. У пациентов с ОП достоверно чаще встречались такие гериатрические синдромы, как СА, гиподинамия и недостаточность питания, а также полипрагмазия, недержание мочи, головокружение. Пациенты с ОП чаще проживали одиноко и использовали вспомогательные средства при передвижении в сравнении с пациентами без ОП. При помощи однофакторного анализа была изучена взаимосвязь между ОП и гериатрическими синдромами (табл. 5).

Проведенный анализ продемонстрировал, что ОП ассоциирован (ОШ от 1.54 до 2.00) с такими гериатрическими синдромами, как СА, гиподинамия, использование вспомогательных средств при передвижении, нарушение сна, сенсорный дефицит по зрению, недержание мочи.

Отдельный интерес представляет ассоциация тяжелого ОП с наличием низкоэнергетических переломов и гериатрических синдромов (табл. 6).
Полученные данные показали, что тяжелый ОП с наличием низкоэнергетических переломов ассоциирован с другими гериатрическими синдромами: ортопедическая патология стопы, депрессия, тревога, хроническая боль. Связь ОП со СА была выявлена вне зависимости от тяжести ОП.

В рамках КГО также оценен функциональный статус пациентов, результаты представлены в табл. 7.

При этом оказалось, что пациенты 1 группы были более хрупкими, чаще имели высокий (5 и более) балл по шкале «Возраст не помеха», зависимость в базовой и инструментальной активности в повседневной жизни, а также низкую скорость ходьбы по сравнению с пациентами 2 группы. Необходимо отметить, что мы не получили достоверных различий в мышечной силе, однако в позиции мышечной функции наблюдались достоверные различия между пациентами обеих групп. Однако при проведении корреляционного анализа у пациентов с ОП выявлена слабая отрицательная корреляция между МПК и временем выполнения теста 5 подъемов 
Таблица 8. Характеристика синдрома падений

\begin{tabular}{|c|c|c|c|c|c|}
\hline & $\begin{array}{c}\text { C OП } \\
(n=178)\end{array}$ & $\%$ & $\begin{array}{l}\text { Без ОП } \\
(n=429)\end{array}$ & $\%$ & $\mathbf{p}$ \\
\hline Количество пациентов с падениями & 93 & 52,2 & 217 & 50,6 & 0,304 \\
\hline Риск падений по шкале Mорсе, баллы & $33,1 \pm 18,7$ & & $33,7 \pm 20,2$ & & 0,158 \\
\hline $\begin{array}{l}\text { Количество пациентов с высоким риском падений } \\
\text { по шкале Морсе }\end{array}$ & 36 & 20,2 & 80 & 18,6 & 0,253 \\
\hline $\begin{array}{l}\text { Риск падений по шкале самооценки риска падений, } \\
\text { баллы }\end{array}$ & $5,87 \pm 3,65$ & & $5,63 \pm 3,3$ & & 0,226 \\
\hline $\begin{array}{l}\text { Количество пациентов с высоким риском падений } \\
\text { по шкале самооценки риска падений }\end{array}$ & 124 & 69,7 & 301 & 70,2 & 0,859 \\
\hline Количество падений за последний год & $2,2 \pm 1,7$ & & $2,1 \pm 1,9$ & & 0,343 \\
\hline Падения в анамнезе & 83 & 89,2 & 161 & 74,2 & 0,009 \\
\hline Страх падений & 40 & 43 & 106 & 48,8 & 0,298 \\
\hline Падения дома & 32 & 34,4 & 64 & 29,5 & 0,292 \\
\hline Исход падений: перелом & 14 & 15,1 & 17 & 7,8 & 0,040 \\
\hline
\end{tabular}

Примечание: Курсивом указаны статистически достоверные результаты.

со стула ( $r=-0,32 ; \mathrm{p}=0,03)$, отражающего мышечную силу в ногах.

Нельзя не акцентировать внимание, что более половины обследуемых пациентов в течение последнего года перенесли падения. В связи с чем нам показалось целесообразным представить отдельно характеристику падений и их последствий (табл. 8).

Из таблицы 8 видно, что у пациентов с ОП в анамнезе чаще имелись указания на падения, а риск повторных падений был высоким. У пациентов 1 группы падения дома встречались несколько чаще. Важно отметить, что у пациентов 1 группы падения завершились переломами в 2 раза чаще, чем во 2 группе. Далее мы проанализировали все случившиеся после 60 лет переломы у пациентов с ОП. Оказалось, что 75 (42,1\%) пациентов с ОП имели низкоэнергетические переломы. Из них 13 (17,3\%) человек - множественные. Наиболее частыми локализациями случившихся переломов были: лучевая кость в типичном месте (29,3\%), тела позвонков (14,7\%), проксимальный отдел бедренной кости (24\%).

\section{ОБСУЖДЕНИЕ}

Репрезентативность выборок

Набор участников исследования проводился только в федеральном научном центре - РГНКЦ. В исследование вошли госпитализированные в гериатрическое отделение пациенты.

Сопоставление с другими публикациями

Распространенность ОП у пациентов, включенных в исследование, составила 29,3\%, что соответствует эпи- демиологическим данным в разных странах мира, в том числе в России и США [2, 28-30]. Целью нашей работы было оценить коморбидный и гериатрический статус пациентов пожилого и старческого возраста с ОП и без него. Как оказалось, у пациентов 1 группы чаще наблюдались заболевания щитовидной железы, болезнь Альцгеймера, болезнь Паркинсона, анемия, остеоартрит, варикозная болезнь. Эти данные имеют подтверждение в литературе. Так, ряд авторов показали в наблюдательных исследованиях и метаанализах, что пациенты с болезнью Паркинсона имеют более высокий риск ОП и низкую МПК по сравнению с контролем. При этом оказалось, что тяжесть ОП коррелирует с тяжестью болезни Паркинсона [31, 32]. Это объясняется тем, что дофаминовые рецепторы экспрессируются в остеобластах и остеокластах и влияют на гомеостаз костей [33]. Связь ОП и болезни Альцгеймера также показана в нескольких исследованиях. Оба заболевания являются многофакторными, включая генетические, метаболические, эндокринные и экологические. Частота переломов у пациентов с болезнью Альцгеймера более чем в три раза превышает частоту переломов у пациентов группы контроля с поправкой на возраст и пол $[34,35]$.

Связь заболеваний щитовидной железы и ОП известна, однако патогенетические механизмы еще обсуждаются. Известно, что хондроциты и остеобласты непосредственно реагируют на гормоны щитовидной железы. Остеокласты также чувствительны к изменениям функции щитовидной железы, однако остается неопределенным, являются ли остеокласты клетками-мишенями или влияние гормонов щитовидной железы на резорбцию кости является косвенным за счет первичного действия гормонов на другие клетки [36, 37]. 
Эпидемиологические данные о распространенности остеоартрита у пациентов с ОП достаточно противоречивы. Ряд авторов демонстрируют, что частота ОП обратно коррелирует с частотой остеоартрита, и эти два заболевания редко сосуществуют совместно у одного человека [38, 39]. Однако исследования последних лет указывают на обратные данные и демонстрируют потерю костной ткани у пациентов с остеоартритом, даже на ранних стадиях заболевания. Достижения в области остеоиммунологии обнаружили влияние цитокинов на кость и обеспечили понимание роли провоспалительных цитокинов и адипокинов в метаболизме хряща и кости [40-42]. Полученные нами данные демонстрируют достоверное увеличение частоты остеоартрита у пациентов с ОП, а также наличие ассоциации между этими заболеваниями (ОШ 4,02; 95\% ДИ 2,65-6, 11; $<<0,05)$.

Необходимо отметить, что большинство заболеваний, которые встречались достоверно чаще у пациентов 1 группы, являются возраст-ассоциированными. Единого механизма, объясняющего их взаимосвязь, на сегодняшний день нет. Наиболее удовлетворяющей является теория хронического системного воспаления. Данная концепция позволяет объяснить более частую встречаемость не только соматических заболеваний, но и гериатрических синдромов у пациентов с ОП. У пациентов 1 группы достоверно чаще встречались СА, недостаточность питания и гиподинамия ( $<<0,001)$. Эти синдромы определяют хрупкость пожилого человека. С другой стороны, возможно и обратное влияние. Многочисленные исследования и метаанализы демонстрируют повышенную секрецию ИЛ-1, ИЛ-6, ФНО при большинстве гериатрических синдромов: СА, саркопении, депрессии, болезни Альцгеймера, хроническом болевом синдроме [43-47]. Повышение провоспалительных цитокинов может обусловливать прогрессию ОП у гериатрических пациентов. Наши данные показывают наличие ассоциации между ОП и СА, гиподинамией, нарушением сна, недержанием мочи.

\section{Клиническая значимость результатов}

Полученные данные демонстрируют, что пациенты с ОП имеют большее число гериатрических синдромов, худший функциональный статус, достоверную связь с возраст-ассоциированными заболеваниями, чаще имеют инвалидность. В связи с чем наличие ОП, даже без ОП переломов, можно предложить рассматривать как маркер хрупкости. Тем более что самая сильная ассоциация ОП выявлена с синдромом СА (ОШ 2,00; 95\% ДИ 1,32-3,04; $p<0,05)$.
Ограничения исследования

Исследование проводилось у пациентов, госпитализированных в гериатрическое отделение стационара. Это выборка пациентов, которые заведомо тяжелее как по соматическому, так и гериатрическому статусу, чем пациенты, наблюдающиеся амбулаторно.

\section{Направления дальнейших исследований}

Считаем целесообразным проведение дальнейших исследований по изучению ассоциации ОП с гериатрическими синдромами, влияния не только на функциональный, но и когнитивный статус пожилых пациентов. Это позволит персонифицированно подходить к определению программы ведения пациентов пожилого и старческого возраста.

\section{ЗАКЛЮЧЕНИЕ}

Пациенты с ОП представляют группу хрупких пациентов с отягощенным соматическим и гериатрическим статусом, что требует особого подхода к их ведению: обязательного проведения комплексной гериатрической оценки, по результатам которой определяется индивидуальный план ведения пожилого пациента.

\section{ДОПОЛНИТЕЛЬНАЯ ИНФОРМАЦИЯ}

Источники финансирования. Работа выполнена по инициативе авторов без привлечения финансирования.

Конфликт интересов. Авторы декларируют отсутствие явных и потенциальных конфликтов интересов, связанных с содержанием настоящей статьи.

Участие авторов. Ховасова Н.О. - существенный вклад в концепцию и дизайн исследования, в получение, анализ данных или интерпретацию результатов, написание статьи; Наумов А.В. - существенный вклад в концепцию и дизайн исследования, в получение, анализ данных или интерпретацию результатов, написание статьи; Ткачева О.Н. существенный вклад в получение, анализ данных или интерпретацию результатов, внесение в рукопись существенной правки с целью повышения научной ценности статьи; Дудинская Е.Н. - существенный вклад в получение, анализ данных или интерпретацию результатов, внесение в рукопись существенной правки с целью повышения научной ценности статьи. Все авторы одобрили финальную версию статьи перед публикацией, выразили согласие нести ответственность за все аспекты работы, подразумевающую надлежащее изучение и решение вопросов, связанных с точностью или добросовестностью любой части работы.

\section{СПИСОК ЛИТЕРАТУРЫ | REFERENCES}

1. NIH Consensus Development Panel on Osteoporosis Prevention, Diagnosis and T. Osteoporosis Prevention, Diagnosis, and Therapy. JAMA J Am Med Assoc. 2001;285(6):785-795 doi: https://doi.org/10.1001/jama.285.6.785

2. Белая Ж.Е., Рожинская Л.Я., Гребенникова Т.А., и др. Краткое изложение проекта федеральных клинических рекомендаций по остеопорозу // Ocmeonopoз и остеопатии. - 2020. - T. 23. №2. - C. 4-21. [Belaya ZE, Rozhinskaya LY, Grebennikova TA, et al. Summary of the draft federal clinical guidelines for osteoporosis. Osteoporosis and Bone Diseases. 2021;23(2):4-21. (In Russ.)]. doi: https://doi.org/10.14341/osteo12710

3. Wright NC, Saag KG, Dawson-Hughes B, et al. The impact of the new National Bone Health Alliance (NBHA) diagnostic criteria on the prevalence of osteoporosis in the USA. Osteoporos Int. 2017;28(4):1225-1232. doi: https://doi.org/10.1007/s00198-016-3865-3.

4. Johnell O, Kanis J. Epidemiology of osteoporotic fractures. Osteoporos Int. 2005;16(S2):S3-S7.

doi: https://doi.org/10.1007/s00198-004-1702-6

5. Dawson A, Dennison E. Measuring the musculoskeletal aging phenotype. Maturitas. 2016;93:13-17. doi: https://doi.org/10.1016/j.maturitas.2016.04.014

6. IOF Compendium of Osteoporosis. 1st ed (2017). Available from: www.iofbonehealth.org

7. Aspray TJ, Hill TR. Osteoporosis and the Ageing Skeleton. Subcell Biochem. 2019;91:453-476. doi: https://doi.org/10.1007/978-981-13-3681-2_16 
8. Haentjens P, Magaziner J, Colon-Emeric CS, et al. Meta-analysis: excess mortality after hip fracture among older women and men. Ann Intern Med. 2010;152(6):380-390. doi: https://doi.org/10.7326/0003-4819-152-6-201003160-00008

9. Stenhagen M, Ekström H, Nordell E, Elmståhl S. Falls in the general elderly population: a 3-and 6-year prospective study of risk factors using data from the longitudinal population study 'Good ageing in Skane.' BMC Geriatr. 2013;13(1):81. doi: https://doi.org/10.1186/1471-2318-13-81

10. Zasadzka E, Borowicz AM, Roszak M, Pawlaczyk M. Assessment of the risk of falling with the use of timed up and go test in the elderly with lower extremity osteoarthritis. Clin Interv Aging. 2015;10:1289-1298. doi: https://doi.org/10.2147/CIA.S86001

11. Bączyk G, Samborski W, Jaracz K. Evaluation of the quality of life of postmenopausal osteoporotic and osteopenic women with or without fractures. Arch Med Sci. 2016;12(4):819-827. doi: https://doi.org/10.5114/aoms.2015.55012

12. Aspray TJ, Hill TR. Osteoporosis and the Ageing Skeleton. Subcell Biochem. 2019;91:453-476. doi: https://doi.org/10.1007/978-981-13-3681-2_16

13. Li G, Thabane L, Papaioannou A, Adachi JD. Comparison between frailty index of deficit accumulation and fracture risk assessment tool (FRAX) in prediction of risk of fractures. Bone. 2015;77:107-114. doi: https://doi.org/10.1016/j.bone.2015.04.028

14. Ensrud KE, Ewing SK, Taylor BC, et al. Frailty and risk of falls, fracture, and mortality in older women: the study of osteoporotic fractures. J Gerontol A Biol Sci Med Sci. 2007;62(7):744-751. doi: https://doi.org/10.1093/gerona/62.7.744

15. Li G, Thabane L, Papaioannou A, loannidis G, et al. An overview of osteoporosis and frailty in the elderly. BMC Musculoskelet Disord. 2017;18(1):46. doi: https://doi.org/10.1186/s12891-017-1403-x

16. Li G, Thabane L, Papaioannou A, Adachi JD. Comparison between frailty index of deficit accumulation and fracture risk assessment tool (FRAX) in prediction of risk of fractures. Bone. 2015;77:107-114. doi: https://doi.org/10.1016/j.bone.2015.04.028

17. Gielen $\mathrm{E}$, Bergmann $\mathrm{P}$, Bruyère $\mathrm{O}$, et al. Osteoporosis in Frail Patients: A Consensus Paper of the Belgian Bone Club. Calcif Tissue Int. 2017;101(2):111-131. doi: https://doi.org/10.1007/s00223-017-0266-3

18. Verschueren S, Gielen E, O'Neill TW, et al. Sarcopenia and its relationship with bone mineral density in middle-aged and elderly European men. Osteoporos Int. 2013;24(1):87-98. doi: https://doi.org/10.1007/s00198-012-2057-z

19. He H, Liu Y, Tian Q, et al. Relationship of sarcopenia and body composition with osteoporosis. Osteoporos Int. 2016;27(2):473-482. doi: https://doi.org/10.1007/s00198-015-3241-8

20. Locquet $M$, Beaudart $C$, Reginster JY, Bruyère $O$. Association Between the Decline in Muscle Health and the Decline in Bone Health in Older Individuals from the SarcoPhAge Cohort. Calcif Tissue Int. 2019;104(3):273-284. doi: https://doi.org/10.1007/s00223-018-0503-4

21. Huo YR, Suriyaarachchi P, Gomez F, et al. Comprehensive nutritional status in sarco-osteoporotic older fallers. J Nutr Health Aging. 2015;19(4):474-480. doi: https://doi.org/10.1007/s12603-014-0543-z

22. Calvani R, Martone AM, Marzetti E, et al. Pre-Hospital Dietary Intake Correlates with Muscle Mass at the Time of Fracture in Older Hip-Fractured Patients. Front Aging Neurosci. 2014;6:269. doi: https://doi.org/10.3389/fnagi.2014.00269

23. Scott D, Seibel M, Cumming R, et al. Does Combined Osteopenia/ Osteoporosis and Sarcopenia Confer Greater Risk of Falls and Fracture Than Either Condition Alone in Older Men? The Concord Health and Ageing in Men Project. Journals Gerontol Ser A. 2019;74(6):827-834. doi: https://doi.org/10.1093/gerona/gly162

24. Tagliaferri C, Wittrant Y, Davicco MJ, et al. Muscle and bone, two interconnected tissues. Ageing Res Rev. 2015;21:55-70. doi: https://doi.org/10.1016/j.arr.2015.03.002

25. Pietschmann P, Mechtcheriakova D, Meshcheryakova A, et al. Immunology of Osteoporosis: A Mini-Review. Gerontology. 2016;62(2):128-137. doi: https://doi.org/10.1159/000431091

26. Franceschi C, Capri M, Monti D, et al. Inflammaging and anti-inflammaging: A systemic perspective on aging and longevity emerged from studies in humans. Mech Ageing Dev. 2007;128(1):92-105. doi: https://doi.org/10.1016/j.mad.2006.11.016

27. Ткачева О.Н., Котовская Ю.В., Рунихина Н.К., и др. Клинические рекомендации «Старческая астения» // Российский журнал гериатрической медииины. — 2020. — №1. - С. 11-46.
[Tkacheva ON, Kotovskaya YV, Runikhina NK, et al. Clinical guidelines on frailty. Russ J Geriatr Med. 2020;128(1):1 1-46. (In Russ.)]. doi: https://doi.org/10.37586/2686-8636-1-2020-11-46

28. Camacho PM, Petak SM, Binkley N, et al. American Association of Clinical Endocrinologists and American College of Endocrinology Clinical Practice Guidelines for the Diagnosis and Treatment of Postmenopausal Osteoporosis - 2016. Endocr Pract. 2016;22:1-42. doi: https://doi.org/10.4158/EP161435.GL

29. Kanis JA, Cooper C, Rizzoli R, Reginster J-Y. European guidance for the diagnosis and management of osteoporosis in postmenopausal women. Osteoporos Int. 2019;30(1):3-44 doi: https://doi.org/10.1007/s00198-018-4704-5

30. Fahimfar N, Noorali S, Yousefi S, et al. Prevalence of osteoporosis among the elderly population of Iran. Arch Osteoporos. 2021;16(1):16 doi: https://doi.org/10.1007/s11657-020-00872-8

31. Gao H, Wei X, Liao J, et al. Lower Bone Mineral Density in Patients with Parkinson's Disease: A Cross-Sectional Study from Chinese Mainland. Front Aging Neurosci. 2015;7:203. doi: https://doi.org/10.3389/fnagi.2015.00203

32. Zhao Y, Shen L, Ji HF. Osteoporosis risk and bone mineral density levels in patients with Parkinson's disease: a meta-analysis. Bone. 2013;52(1):498-505. doi: https://doi.org/10.1016/j.bone.2012.09.013

33. Handa K, Kiyohara S, Yamakawa T, et al. Bone loss caused by dopaminergic degeneration and levodopa treatment in Parkinson's disease model mice. Sci Rep. 2019;9(1):13768. doi: https://doi.org/10.1038/s41598-019-50336-4

34. Buchner DM. Falls and Fractures in Patients With Alzheimer-Type Dementia. JAMA. 1987;257(11):1492-1495. doi: https://doi.org/10.1001/jama.1987.03390110068028

35. Downey $\mathrm{CL}$, Young A, Burton EF, et al. Dementia and osteoporosis in a geriatric population: Is there a common link? World J Orthop. 2017;8(5):412-423. doi: https://doi.org/10.5312/wjo.v8.i5.412.

36. Bassett JH, Williams GR. Role of Thyroid Hormones in Skeletal Development and Bone Maintenance. Endocr Rev. 2016;37(2):135-187. doi: https://doi.org/10.1210/er.2015-1106

37. Williams GR, Bassett JHD. Thyroid diseases and bone health. J Endocrinol Invest. 2018:41(1):99-109. doi: https://doi.org/10.1007/s40618-017-0753-4

38. Stewart A, Black AJ. Bone mineral density in osteoarthritis. Curr Opin Rheumatol. 2000;12(5):464-467. doi: https://doi.org/10.1097/00002281-200009000-00021

39. Dequeker J, Aerssens J, Luyten FP. Osteoarthritis and osteoporosis: clinical and research evidence of inverse relationship. Aging Clin Exp Res. 2003;15(5):426-439. doi: https://doi.org/10.1007/BF03327364

40. Lingard EA, Mitchell SY, Francis RM, et al. The prevalence of osteoporosis in patients with severe hip and knee osteoarthritis awaiting joint arthroplasty. Age Ageing. 2010;39(2):234-239. doi: https://doi.org/10.1093/ageing/afp222

41. Bultink IEM, Lems WF. Osteoarthritis and Osteoporosis: What Is the Overlap? Curr Rheumatol Rep. 2013;15(5):328. doi: https://doi.org/10.1007/s11926-013-0328-0

42. Domingues VR, de Campos GC, Plapler PG, de Rezende MU. Prevalence of osteoporosis in patients awaiting total hip arthroplasty. Acta Ortop Bras. 2015;23(1):34-37. doi: https://doi.org/10.1590/1413-78522015230100981

43. Soysal P, Stubbs B, Lucato P, et al. Inflammation and frailty in the elderly: A systematic review and meta-analysis. Ageing Res Rev. 2016;31:1-8. doi: https://doi.org/10.1016/j.arr.2016.08.006

44. Ma L, Sha G, Zhang Y, Li Y. Elevated serum IL-6 and adiponectin levels are associated with frailty and physical function in Chinese older adults. Clin Interv Aging. 2018;13:2013-2020. doi: https://doi.org/10.2147/CIA.S180934

45. Bano G, Trevisan C, Carraro S, et al. Inflammation and sarcopenia: A systematic review and meta-analysis. Maturitas. 2017;96:10-15. doi: https://doi.org/10.1016/j.maturitas.2016.11.006

46. Ng A, Tam WW, Zhang MW, et al. IL-1 $\beta, I L-6$, TNF- a and CRP in Elderly Patients with Depression or Alzheimer's disease: Systematic Review and Meta-Analysis. Sci Rep. 2018;8(1):12050. doi: https://doi.org/10.1038/s41598-018-30487-6

47. Zille de Queiroz B, de Britto Rosa NM, Pereira DS, et al. Inflammatory mediators and the risk of falls among older women with acute low back pain: data from Back Complaints in the Elders (BACE) — Brazil. Eur Spine J. 2020;29(3):549-555. doi: https://doi.org/10.1007/s00586-019-06168-x 
Рукопись получена: 18.04.2021. Одобрена к публикации: 15.06.2021. Опубликована online: 30.06.2021.

\section{ИНФОРМАЦИЯ ОБ АВТОРАХ [AUTHORS INFO]}

*Ховасова Наталья Олеговна, к.м.н., доцент [Natalia O. Khovasova, PhD, associate Professor]; адрес: г. Mосква, ул. 1-я Леонова, д. 16 [Address: Moscow, First Leonova str., 16]; ORCID: https://orcid.org/0000-0002-3066-4866; eLibrary SPIN: 7387-7710; e-mail: natashahov@mail.ru

Наумов Антон Вячеславович, д.м.н., доцент [Anton V. Naumov, PhD]; ORCID: https://orcid.org/0000-0002-6253-621X; eLibrary SPIN: 4763-9738; e-mail: nanton78@gmail.com

Ткачева Ольга Николаевна, д.М.н. професcop [Olga N. Tkacheva, PhD]; ORCID: https://orcid.org/0000-0002-4193-688X; eLibrary SPIN: 6129-5809; e-mail: ton@rgnkc.ru

Дудинская Екатерина Наильевна, К.M.H. [Ekaterina N. Dudinskaya, PhD]; ORCID: https://orcid.org/0000-0001-7891-6850; eLibrary SPIN: 4985-6315; e-mail: katharina.gin@gmail.com

\section{ЦИТИРОВАТЬ}

Ховасова Н.О., Наумов А.В., Ткачева О.Н., Дудинская Е.Н. Характеристика гериатрического и соматического статуса у пациентов с остеопорозом // Проблемы эндокринологии. — 2021. — Т. 67. — № 3. — С. 45-54. doi: https://doi.org/10.14341/probl12751

\section{TO CITE THIS ARTICLE:}

Khovasova NO, Naumov AV, Tkacheva ON, Dudinskaya EN. Characteristics of geriatric and somatic status in patients with osteoporosis. Problems of Endocrinology. 2021;67(3):45-54. doi: https://doi.org/10.14341/probl12751 